\title{
Sentinel-1 Image Matching Using Strong Scatters
}

\author{
M. A. Ghannadi a *, M. Saadatseresht ${ }^{\mathrm{a}}$, M. Motagh ${ }^{\mathrm{b}}$ \\ ${ }^{a}$ Dept. of surveying and Geomatics engineering, University of Tehran, Tehran, Iran \\ (amin.ghannadi, msaadat@ut.ac) \\ ${ }^{\mathrm{b}}$ Department of Earthquake Risk and Early Warning, GeoForschungs Zentrum, Potsdam, Germany \\ motagh@gfz-potsdam.de
}

KEY WORDS: SAR image matching, Santinel-1, strong scatter, multi quadric, least square matching

\begin{abstract}
:
The availability of new radar spaceborne sensors offers new interesting potentialities for the geomatics application: spatial and temporal change detection, generation of Digital Elevation Model(DEM) using radargrametry and interferometry. Since the start of the sentinel-1 mission to take images from different regions all over the world, the ability to use these images in variety domains has been treasured. This paper suggests a method for image matching using strong scatters. all the experiments are done on sentinel-1 stereo images from Jam, Bushehr, Iran.
\end{abstract}

\section{INTRODUCTION}

Image matching is one of the main stages in radargrammetry, interferometry and change detection. The getting better in SAR image matching result has always been a challenge that researchers have been trying to overcome. SAR image matching is more ambiguous compared to optical image matching. Generally, the following two reasons can be an explanation of the matter: the existence of geometric phenomenon and also radiometric noise in SAR imaging. these geometric difficulties consist of shadow, layover, and foreshortening, which cause challenge in the structure of SAR images in respect to other imageries[1]. nature of SAR imaging systems causes an interference pattern called speckle. speckle is modeled as a multiplicative noise, which degrades the images[3].

The European Space Agency (ESA) satellite, sentinel-1 was launched in april 3, 2014 and images have been in users access since october 3, 2014[4]. the imagery is derived from SAR systems so one of its main characteristics is bright points which are demonstrated because of radar waves back scattered from urban areas or mountains. previously these "point-like targets" have been used in order to achieve image offset or interferometry[5]. the main aim in this paper is image matching using point-like targets as points with high reliability.

\section{METHODOLOGY}

The suggested method includes 4 stages:

1. Due to the presence of intrinsic speckle noise in SAR images, at the first, a wiener filter is used to reduce noise and then the matching is done.

2. Using template and calculating cross correlation for all the points existing so image targets or strong scatters will be extracted.
3. Assigning features to extracted points so strong scatters will be matched to one another in two images. these matched points are going to be used in the next stage.

4. Using multiquadric equations for image matching. matched points in stage 2 are used and the precision will be around one pixel.

$\left[\begin{array}{l}d X_{1} \\ d X_{2} \\ d X_{3} \\ \cdot \\ d X_{n}\end{array}\right]=\left[\begin{array}{cccc}D_{11} & D_{12} & \cdot & D_{1 n} \\ D_{21} & D_{22} & \cdot & D_{2 n} \\ \cdot & \cdot & \cdot & \cdot \\ D_{n 1} & D_{n 2} & \cdot & D_{n n}\end{array}\right]\left[\begin{array}{l}\lambda_{1} \\ \lambda_{2} \\ \lambda_{3} \\ \cdot \\ \lambda_{n}\end{array}\right]\left[\begin{array}{l}d Y_{1} \\ d Y_{2} \\ d Y_{3} \\ \cdot \\ d Y_{n}\end{array}\right]=\left[\begin{array}{cccc}D_{11} & D_{12} & \cdot & D_{1 n} \\ D_{21} & D_{22} & \cdot & D_{2 n} \\ \cdot & \cdot & \cdot & \cdot \\ D_{n 1} & D_{n 2} & \cdot & D_{n n}\end{array}\right]\left[\begin{array}{l}\beta_{1} \\ \beta_{2} \\ \beta_{3} \\ \cdot \\ \beta_{n}\end{array}\right]$

$\mathrm{dx}$ and dy in equation (1) show the planar shift between matched points from stage 2. D is the distance matrix for $n$ strong scatter points in first image. $\lambda$ and $\beta$ coefficients in equation (2) will be used to find matched points for selected points in first image.

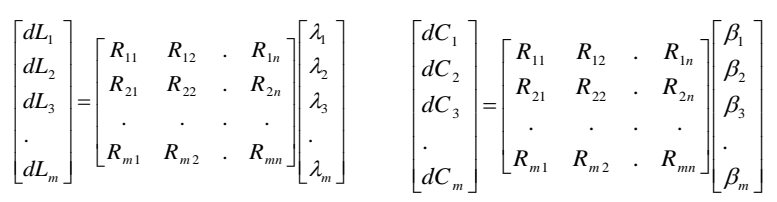

$\mathrm{R}$ matrix in equation (2) is the distance matrix between $\mathrm{n}$ strong scatterer points and $\mathrm{m}$ selected points. $\mathrm{dL}$ and $\mathrm{dC}$ will show the displacement between matched points and selected points[2].

The output will be a coarse matching and its precision is about one pixels.

The general workflow proposed method is shown in figure 1

\footnotetext{
* Corresponding author
} 
The International Archives of the Photogrammetry, Remote Sensing and Spatial Information Sciences, Volume XL-1/W5, 2015 International Conference on Sensors \& Models in Remote Sensing \& Photogrammetry, 23-25 Nov 2015, Kish Island, Iran

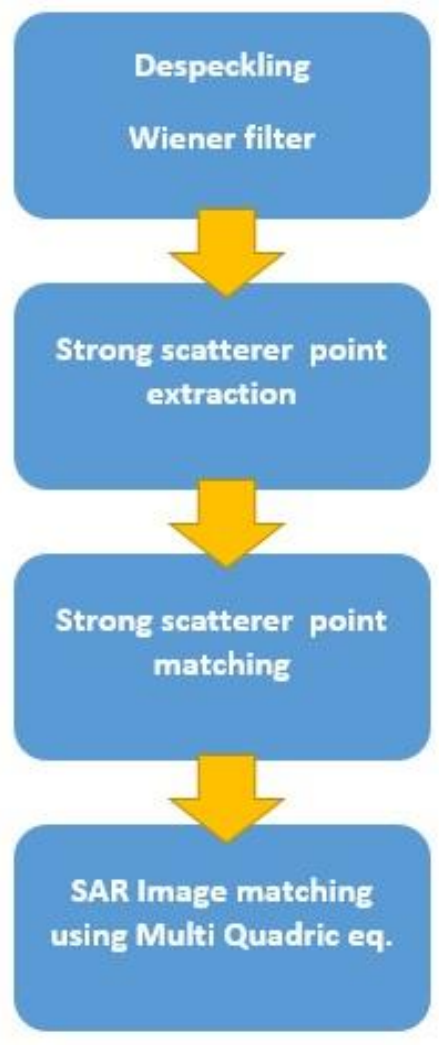

Figure 1. General workflow of proposed method

\section{DATA AND EXPERIMENTS}

All the experiments are done on sentinel-1 stereo images from Jam, Bushehr, Iran. the images are taken in C-band and VV polarization in 2015.04.17 and 2015.05.11 (Shown in figure 2).
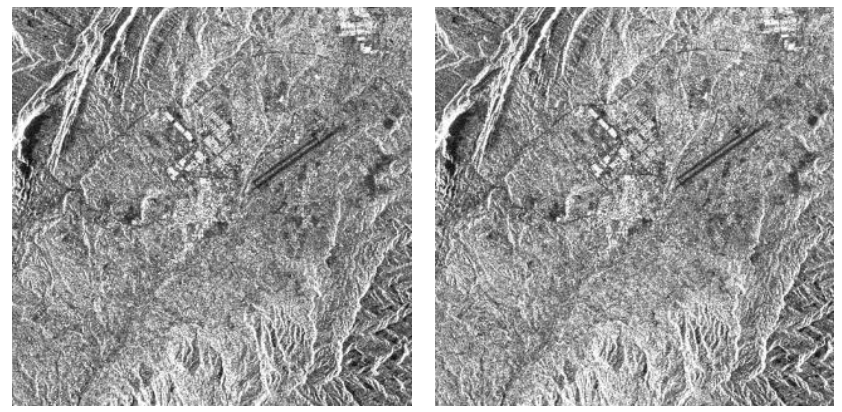

Figure 2. Sentinel-1 stereo images of JAM-Bushehr-Iran

At first, to reduce speckle noise effect on image, wiener filter is used. after this, point like target are extracted using tempelate. the template and some point like target on images are shown in figure 3. then extracted points are match on two images. in this paper, 10 of the best match points are selected for next step. this points are shown in figure 4. next step is selecting optional points on master image and gaining corresponding points on slave image using multi quadric equation and point like target match points.

To do the experiment a $30 \times 30$ optional grid of points are chosen on the first image. then, using suggested method, it will result in matched points in second image.
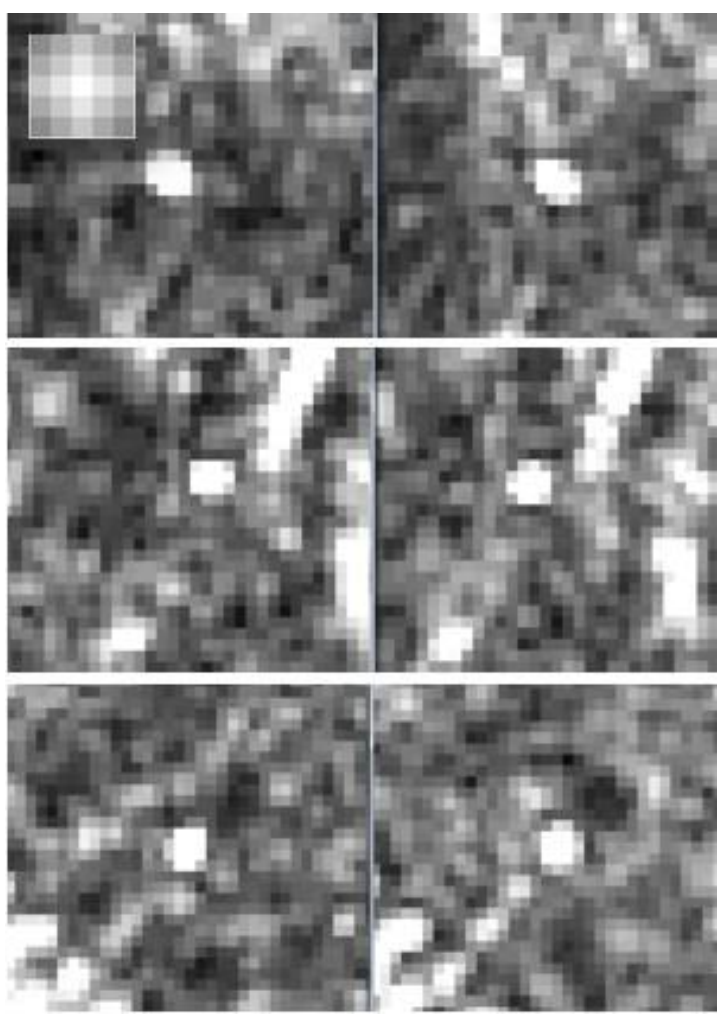

Figure 3. template and some point like target on images

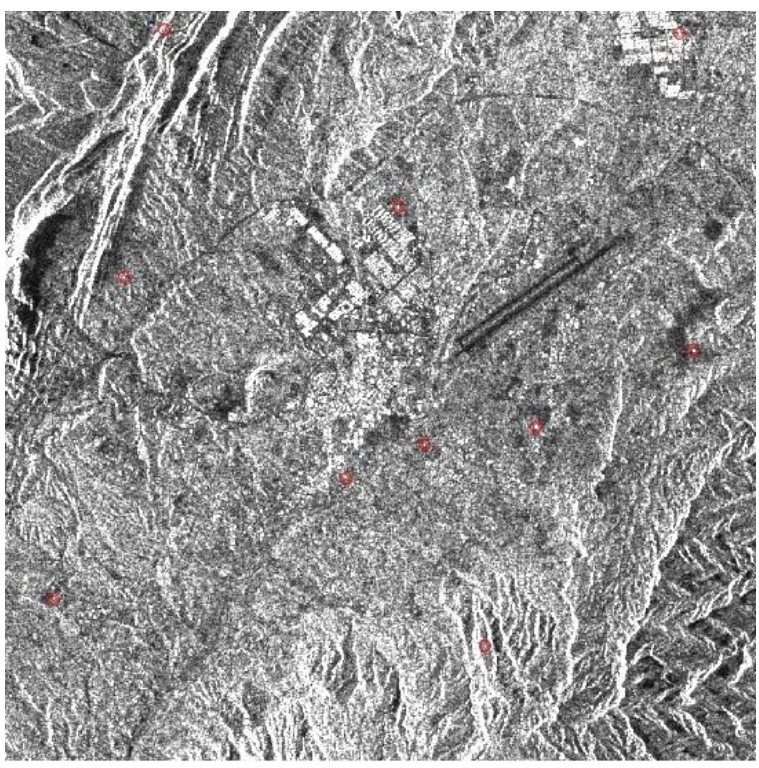

Figure 4. 10 of the best match points on master image

Evaluation factor for matching accuracy is manual measurement for random points of grid. then 50 points are selected randomly and accuracy of matching process are observed.

The result show that 43 points have one pixel diffrence with the corresponding point and the rest have two pixels diffrence with the corresponding point. grid of points on master image and matched point on slave image are shown in figure 5 . 

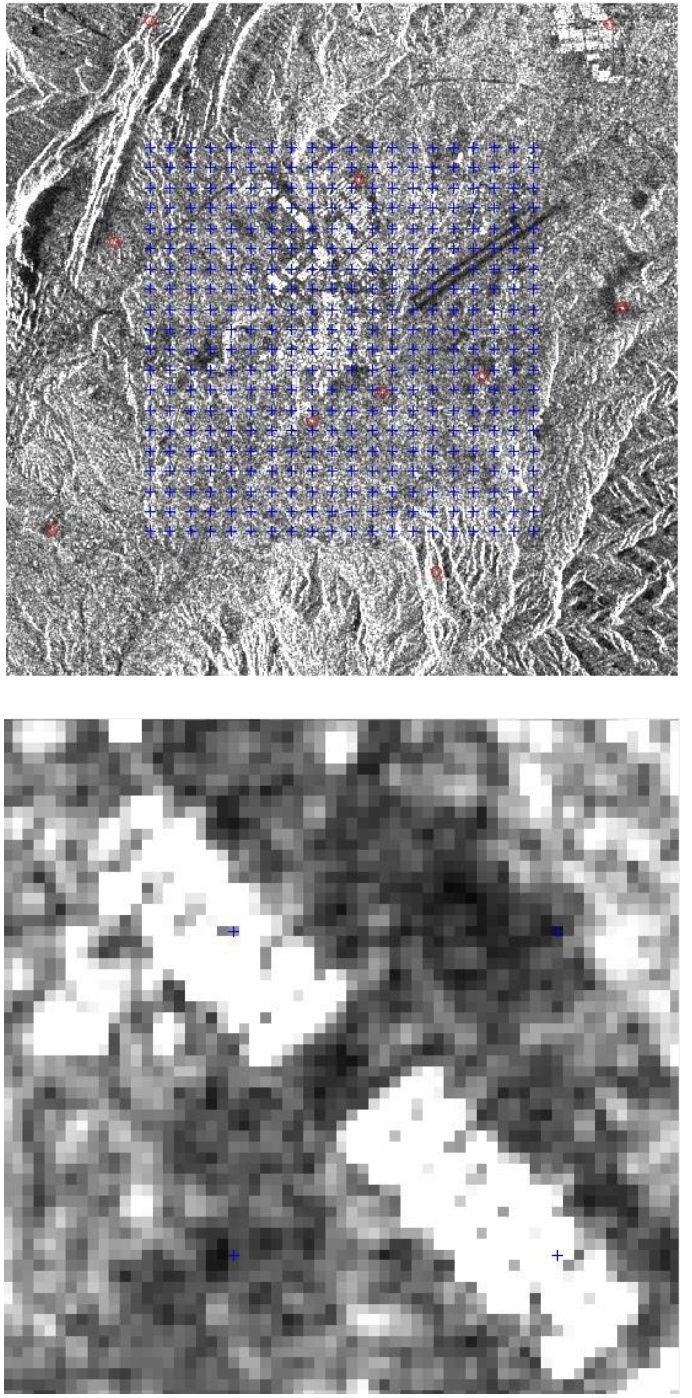
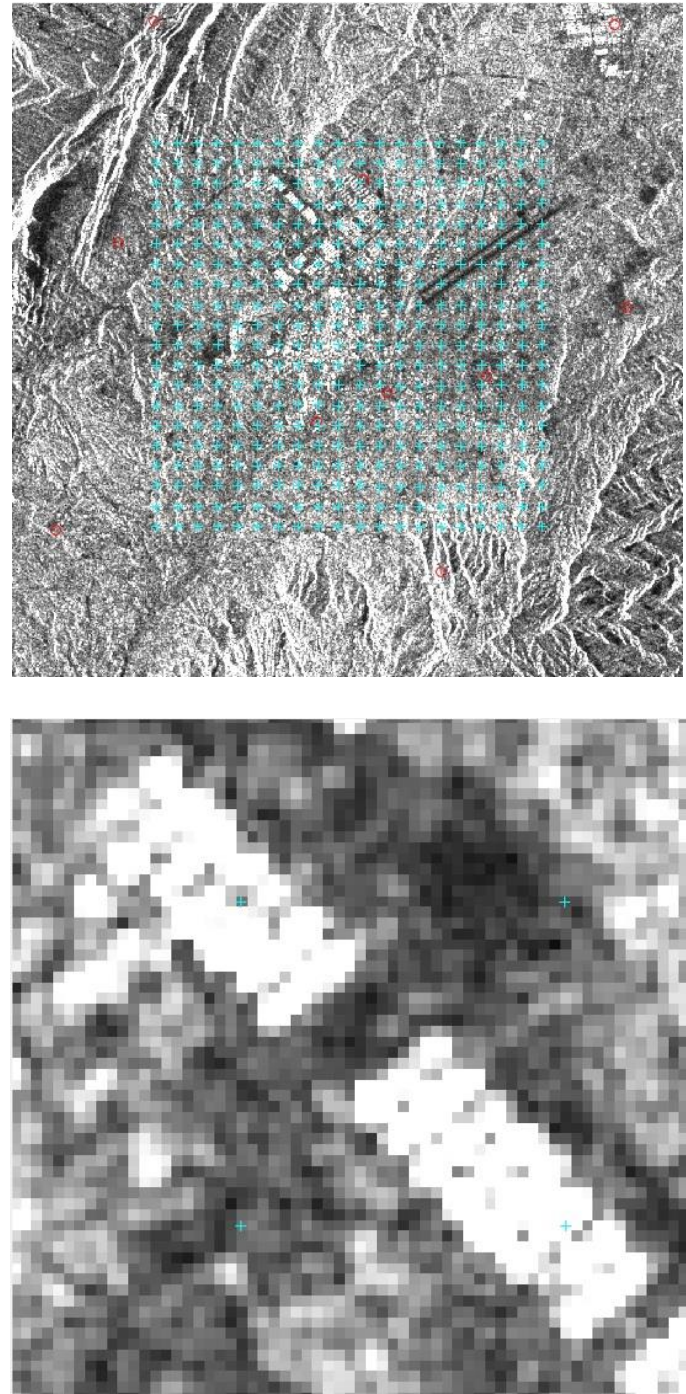

Figure 5. grid of points on master image(up and left), matched points on slave image(up and right). and sample of some matched points(down)

\section{CONCLUSION}

Strong scatterer points have high reliability and can be used as base points in this method. In other words, these points ensure accuracy in the algorithm.in this paper shows that $86 \%$ of matched points have accuracy with one pixel. this method will be used for both sparse and dense matching which means using all pixels in the image. the advantage of this method is high accurcy and the main disadvantage of it is time-consuming process stage and large amounts of calculations.

\section{REFERENCES}

[1] Capaldo, P., et al., 2015, "Evaluation and comparison of different radargrammetric approaches for Digital Surface Models generation from COSMO-SkyMed, TerraSAR-X, RADARSAT-2 imagery: Analysis of Beauport (Canada) test site." ISPRS Journal of Photogrammetry and Remote Sensing 100: 60-70.

[2] Ghannadi, M, A, et al., 2013, "Using Multi Quadric Equation and Least Square Matching Method for SAR Image Matching." GEJ. 4 (4) :1-12
[3] Ghannadi, M. A., et al., 2013, "Using Multi Resolution Census and Ranklet Transformation in Long Base Line SAR Image Matching." ISPRS-International Archives of the Photogrammetry, Remote Sensing and Spatial Information Sciences 1.3, 181-184.

[4] Geudtner, Dirk, et al., 2014, "Sentinel-1 System capabilities and applications." Geoscience and Remote Sensing Symposium (IGARSS), IEEE International. IEEE, 2014.

[5] Hu, Xie, Teng Wang, and Mingsheng Liao., 2014, "Measuring coseismic displacements with point-like targets offset tracking." Geoscience and Remote Sensing Letters, IEEE 11.1, 283-287. 